\title{
Hawking Radiation of Apparent Horizon in a FRW Universe
}

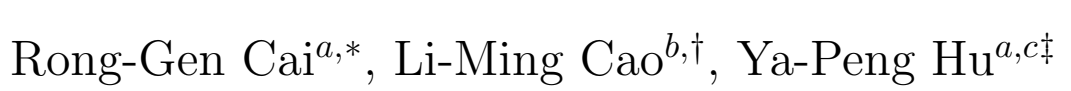 \\ a Institute of Theoretical Physics, Chinese Academy of Sciences, \\ P.O. Box 2735, Beijing 100190, China \\ ${ }^{b}$ Asia Pacific Center for Theoretical Physics, Pohang, Gyeongbuk 790-784, Korea \\ ${ }^{c}$ Graduate School of the Chinese Academy of Sciences, Beijing 100039, China
}

\begin{abstract}
Hawking radiation is an important quantum phenomenon of black hole, which is closely related to the existence of event horizon of black hole. The cosmological event horizon of de Sitter space is also of the Hawking radiation with thermal spectrum. By use of the tunneling approach, we show that there is indeed a Hawking radiation with temperature, $T=1 / 2 \pi \tilde{r}_{A}$, for locally defined apparent horizon of a Friedmann-Robertson-Walker universe with any spatial curvature, where $\tilde{r}_{A}$ is the apparent horizon radius. Thus we fill in the gap existing in the literature investigating the relation between the first law of thermodynamics and Friedmann equations, there the apparent horizon is assumed to have such a temperature without any proof. In addition, we stress the implication of the Hawking temperature associated with the apparent horizon.
\end{abstract}

\footnotetext{
*e-mail address: cairg@itp.ac.cn

$\dagger$ e-mail address: caolm@apctp.org

${ }_{\ddagger}^{\ddagger}$ e-mail address: yapenghu@itp.ac.cn
} 
Introduction: A remarkable feature of black hole is the existence of event horizon. The event horizon can be viewed as boundary of black hole. It is the existence of event horizon so that Hawking [1] found that black hole behaves like a black body, emitting thermal radiation, with a temperature proportional to its surface gravity on the event horizon and Bekenstein argued [2] that there is an entropy proportional to its horizon area for a black hole. The Hawking temperature and horizon entropy together with the black hole mass obey the first law of black hole thermodynamics [3]. Hawking radiation and black hole entropy help us to deepen our understanding on properties of quantum gravity, although a completely self-consistent quantum theory of gravity is still not yet available so far. A seminal work relevant to Hawking radiation of black hole is done by Unruh [4], who found that an uniformly accelerating observer with acceleration $a$ in Minkowskian spacetime can detect a thermal spectrum with temperature $T=a / 2 \pi$. Throughout the paper we use the geometric unit where $c=\hbar=1$, but we explicitly write down the gravitational constant $G$. The Unruh radiation is closely related to the existence of Rindler causal horizon for the observer. In addition, Gibbons and Hawking in 1977 showed [5] that very similar to black hole horizon, there is also a Hawking radiation with temperature $T=1 / 2 \pi l$ associated with cosmological event horizon in de Sitter space, here $l$ is the horizon radius of de Sitter space. The existence of the cosmological event horizon is particularly clear in the static coordinates of de Sitter space, which is

$$
d s^{2}=-\left(1-\frac{r^{2}}{l^{2}}\right) d t^{2}+\left(1-\frac{r^{2}}{l^{2}}\right)^{-1} d r^{2}+r^{2} d \Omega_{2}^{2}
$$

The cosmological event horizon locates at $r=l$. The Hawking temperature $T=1 / 2 \pi l$ is measured by a comoving observer at $r=0$. Note that event horizon is a global concept for a spacetime. Therefore, locally it is not known whether there is an event horizon associated with a certain dynamical (time-dependent) spacetime at some time. Thus this causes the difficulty to discuss Hawking radiation for a dynamic black hole. More recently Hawyard et al. [6] have attacked this issue. Using Hamilton-Jacobi equation of particles to discuss Hawking radiation of stationary black hole, see also [7].

Assuming there is a proportionality between entropy and horizon area, Jacobson [8] derived the Einstein field equation by using the fundamental Clausius relation, $\delta Q=T d S$, connecting heat, temperature and entropy. The key idea is to demand that this relation holds for all the local Rindler causal horizon through each spacetime point, with $\delta Q$ and $T$ interpreted as the energy flux and Unruh temperature seen by an accelerated observer just inside the horizon. In this way, Einstein field equation is nothing, but an equation of state of spacetime. Applying this idea to $f(R)$ theory [9, 10, 11] and scalar- 
tensor theory [10, 12, it turns out that a nonequilibrium thermodynamic setup has to be employed. For another viewpoint, see [13, 14].

It is quite interesting to note that assuming the apparent horizon of a FriedmannRobertson-Walker (FRW) universe has temperature $T$ and entropy $S$ satisfying

$$
T=\frac{1}{2 \pi \tilde{r}_{A}}, \quad S=\frac{A}{4 G},
$$

where $\tilde{r}_{A}$ is the radius of the apparent horizon and $A$ is the area of the apparent horizon, one is able to derive Friedmann equations of the FRW universe with any spatial curvature by applying the Clausius relation to apparent horizon [15]. This works not only in Einstein gravitational theory, but also in Gauss-Bonnet and Lovelock gravity theories. Here a key ingredient is to replace the entropy area formula in Einstein theory by using entropy expressions of black hole horizon in those higher order curvature theories. For related discussions see also [16]. These results should closely relate to the fact that Einstein field equation can be rewritten as an unified first law [17]. Indeed, at the apparent horizon of FRW universe, the first Friedmann equation can be cast to a universal form like the first law of thermodynamics [12, 18]. Note that in those works the apparent horizon is also assumed to have a temperature $T=\kappa / 2 \pi$ without any proof, here $\kappa$ is the surface gravity of apparent horizon. This form also holds in RSII brane world scenario, warped DGP model and even more complicated case with a Gauss-Bonnet term in bulk [19]. Based on this, one is able to find the relation between entropy expression of apparent horizon and horizon geometry in brane world scenarios. These results have been summarized in [20]. For further discussions in this direction see [21]. On the other hand, there also exist some studies in the relation between Einstein field equation and first law of thermodynamics in the setup of black hole spacetime [22].

However, a crucial problem remains in those investigations relating Friedmann equation to the first law of thermodynamics. That is, is there indeed a Hawking radiation with temperature $T=1 / 2 \pi \tilde{r}_{A}$ associated with the apparent horizon of the FRW universe since in those studies it is assumed without strict proof? Which observer sees the Hawking temperature? In the present paper we are going to fill in this gap. By applying the Hamilton-Jacobi method [7] and Parikh-Wilczek approach [23], which are initially designed to study the Hawking radiation of stationary black hole as a tunneling process of particle, we show that as in the case of black hole horizon, there is indeed a thermal radiation for the apparent horizon of FRW universe.

Hamilton-Jacobi Method: Let us start with the FRW metric

$$
d s^{2}=-d t^{2}+a^{2}(t)\left(\frac{d r^{2}}{1-k r^{2}}+r^{2} d \Omega_{2}^{2}\right),
$$


where $t$ is the cosmic time, $r$ is the comoving coordinate, $a$ is the scale factor, $d \Omega_{2}^{2}$ denotes the line element of a 2-dimensional sphere with unit radius, $k=1,0$ and -1 represent a closed, flat and open FRW universe, respectively. Define $\tilde{r}=a r$, the metric (3) can be rewritten as $d s^{2}=h_{a b} d x^{a} d x^{b}+\tilde{r}^{2} d \Omega_{n}^{2}$, where $x^{a}=(t, r), h_{a b}=\operatorname{diag}\left(-1, a^{2} /\left(1-k r^{2}\right)\right)$. Without the whole evolution history of the universe, one cannot know whether there is a cosmological event horizon. However, apparent horizon always exists in the FRW universe since it is a local quantity of spacetime. By definition, $h^{a b} \partial_{a} \tilde{r} \partial_{b} \tilde{r}=0$, we can know the location of the apparent horizon in the FRW universe, $\tilde{r}=\tilde{r}_{A} \equiv 1 / \sqrt{H^{2}+k / a^{2}}$. Here $H=\dot{a} / a$ is the Hubble parameter. When $k=0$, the apparent horizon is just the Hubble horizon. We see that for a de Sitter space where $H$ is a constant and cosmological event horizon radius is $H^{-1}$, the apparent horizon and cosmological event horizon coincide with each other only in the case of $k=0$.

In order to discuss the tunneling of particle, it turns out convenient to use the coordinates $(t, \tilde{r})$. In that case, the metric (3) can be rewritten as

$$
d s^{2}=-\frac{1-\tilde{r}^{2} / \tilde{r}_{A}^{2}}{1-k \tilde{r}^{2} / a^{2}} d t^{2}-\frac{2 H \tilde{r}}{1-k \tilde{r}^{2} / a^{2}} d t d \tilde{r}+\frac{1}{1-k \tilde{r}^{2} / a^{2}} d \tilde{r}^{2}+\tilde{r}^{2} d \Omega_{2}^{2} .
$$

We note that when $k=0$, the metric is quite similar to the Painlevé-de Sitter metric for the de Sitter space [24], where $\tilde{r}_{A}=H^{-1}=l$ is a constant. For the metric (4), the corresponding Kodama vector [25] is

$$
K^{a} \equiv-\epsilon^{a b} \nabla_{b} \tilde{r}=\sqrt{1-k \tilde{r}^{2} / a^{2}}(\partial / \partial t)^{a}
$$

where $\epsilon_{a b}=\frac{1}{\sqrt{1-k \tilde{r}^{2} / a^{2}}}(d t)_{a} \wedge(d \tilde{r})_{b}$. Thus one has $K_{a} K^{a}=-\left(1-\tilde{r}^{2} / \tilde{r}_{A}^{2}\right)$. Therefore the Kodama vector is time-like, null and space-like as $\tilde{r}<\tilde{r}_{A}, \tilde{r}=\tilde{r}_{A}$ and $\tilde{r}>\tilde{r}_{A}$, respectively. Note that the Kodama vector is very similar to the Killing vector $(\partial / \partial t)^{a}$ in the de Sitter space (1), the latter is time-like, null and space-like when $r<l, r=l$ and $r>l$, respectively. Note that the existence of the Kodama vector will play a crucial role in our discussion.

Before preceding, let us stress some differences between stationary black hole spacetime and time-dependent dynamical FRW spacetime. For stationary black hole spacetime, one can define a time-like Killing vector. By the time-like Killing vector, one is able to obtain a conserved mass (energy) associated with the stationary black hole spacetime. Also one can define a conserved energy of a particle moving in the stationary black hole spacetime. On the other hand, there is no time-like Killing vector in the dynamical FRW spacetime. But the Kodama vector defined in (5) could play a similar role in the FRW spacetime as the time-like Killing vector does in the stationary black hole spacetime. By the Kodama 
vector, one can define a conserved quantity [26], Misner-Sharp energy [27], for the FRW spacetime, which plays a crucial role in investigating the relation between the first law and Friedmann equations [12, 18]. By the time-like Kodama vector inside the apparent horizon, one can also therefore define a conserved energy of a particle moving in the FRW spacetime, very like the case for the time-like Killing vector in the stationary black hole spacetime. This point is crucial in the following discussion.

Following the discussion for a dynamical black hole [6], we consider a particle with mass $m$ radially moving in the background (44). The Hamilton-Jacobi equation is

$$
g^{\mu \nu} \partial_{\mu} \mathbf{S} \partial_{\nu} \mathbf{S}+m^{2}=0
$$

By use of the Kodama vector (5), one therefore can define the energy $\omega$ and radial momentum $k_{\tilde{r}}$ associated with the particle

$$
\omega=-K^{a} \partial_{a} \mathbf{S}=-\sqrt{1-k \tilde{r}^{2} / a^{2}} \partial_{t} \mathbf{S}, \quad k_{\tilde{r}}=(\partial / \partial \tilde{r})^{a} \partial_{a} \mathbf{S}=\partial_{\tilde{r}} \mathbf{S} .
$$

Thus the action $\mathbf{S}$ can be written as

$$
\mathbf{S}=-\int \frac{\omega}{\sqrt{1-k \tilde{r}^{2} / a^{2}}} d t+\int k_{\tilde{r}} d \tilde{r}
$$

Substituting the action into the Hamilton-Jacobi equation, one has

$$
-\frac{\omega^{2}}{1-k \tilde{r}^{2} / a^{2}}+\frac{2 H \tilde{r} \omega}{\sqrt{1-k \tilde{r}^{2} / a^{2}}} k_{\tilde{r}}+\left(1-\frac{\tilde{r}^{2}}{\tilde{r}_{A}^{2}}\right) k_{\tilde{r}}^{2}+m^{2}=0
$$

which has solutions

$$
k_{\tilde{r}}=\frac{-H \tilde{r} \pm \sqrt{H^{2} \tilde{r}^{2}+\left(1-\tilde{r}^{2} / \tilde{r}_{A}^{2}\right)\left[1-m^{2}\left(1-k \tilde{r}^{2} / a^{2}\right) / \omega^{2}\right]}}{\left(1-\tilde{r}^{2} / \tilde{r}_{A}^{2}\right) \sqrt{1-k \tilde{r}^{2} / a^{2}}} \omega
$$

where the plus/minus sign corresponds to an outgoing/incoming mode. Now we consider an incoming mode since the observer is inside the apparent horizon, like the case of particle tunneling for the cosmological event horizon in de Sitter space [24]. It is obvious that the action $\mathbf{S}$ has a pole at the apparent horizon. Through a contour integral, we obtain an imaginary part of the action

$$
\begin{aligned}
\operatorname{Im} \mathbf{S} & =-\operatorname{Im} \int \frac{H \tilde{r}+\sqrt{H^{2} \tilde{r}^{2}+\left(1-\tilde{r}^{2} / \tilde{r}_{A}^{2}\right)\left[1-m^{2}\left(1-k \tilde{r}^{2} / a^{2}\right) / \omega^{2}\right]}}{\left(1-\tilde{r}^{2} / \tilde{r}_{A}^{2}\right) \sqrt{1-k \tilde{r}^{2} / a^{2}}} \omega d \tilde{r} \\
& =\pi \tilde{r}_{A} \omega .
\end{aligned}
$$


Note from (10) that in the coordinate (4), there is no contribution of an outgoing particle to the imaginary part of the action, as in the case of an ingoing particle in the black hole spacetime [7, 23]. In the WKB approximation, the emission rate $\Gamma$ is the square of the tunneling amplitude (here the particle tunnels from outside to inside the apparent horizon)

$$
\Gamma \propto \exp (-2 \operatorname{Im} \mathbf{S})
$$

Combining (12) with (11), one can see clearly that the emission rate can be cast in a form of thermal spectrum, $\Gamma \sim \exp (-\omega / T)$, with temperature

$$
T=\frac{1}{2 \pi \tilde{r}_{A}} .
$$

Thus we have finished the proof that an observer inside the apparent horizon will see a thermal spectrum with temperature (13) when particles tunnel from outside the apparent horizon to inside the apparent horizon. This can be explained as Hawking radiation of apparent horizon in the same spirit in the tunneling approach proposed by Parikh and Wilczek that the Hawking radiation of black hole is expressed as a tunneling phenomenon. Furthermore, at this level of approximation, the mass of particle does not enter the emission rate. This is just the remarkable feature of thermal spectrum. In addition, let us stress here that since the energy $\omega$ is measured by the observer with the Kodama vector (5), the thermal spectrum is therefore seen by the same observer. That is to say, the Hawking temperature (13) is measured by the Kodama observer inside the apparent horizon.

Tunneling of Massless Particle: Next we further show that one can indeed assign the temperature (13) to the apparent horizon of the FRW universe by following the standard approach of massless particle tunneling across the de Sitter horizon [24]. The basic idea is the same as the above, in the semiclassical approximation (WKB approximation), the emission rate can be related to the imaginary part of action of a system. As in the case of de Sitter space [24], we will consider the s-wave emission of massless particle. Higher partial wave emission is suppressed by $\hbar$. In the s-wave approximation, particles can be viewed as massless shells, and move along a radial null geodesic.

The radial null geodesic for the metric (4) obeys

$$
\dot{\tilde{r}}=H \tilde{r} \pm \sqrt{H^{2} \tilde{r}^{2}+\left(1-\tilde{r}^{2} / \tilde{r}_{A}^{2}\right)}
$$

where the plus/minus sign corresponds to an outgoing/incoming null geodesic. We consider an incoming geodesic since the particles tunnel from outside to inside the apparent horizon. In addition, let us note that we are only interested in the imaginary part of 
action, therefore we need only to calculate the imaginary part produced by the tunneling particles since remaining part is always real. The imaginary part is produced by particles tunneling through a barrier, the classically forbidden region. The imaginary part can be obtained as follows.

$$
\operatorname{Im} \mathbf{S}=\operatorname{Im} \int_{\tilde{r}_{i}}^{\tilde{r}_{f}} p_{\tilde{r}} d \tilde{r}=\operatorname{Im} \int_{\tilde{r}_{i}}^{\tilde{r}_{f}} \int_{0}^{p_{\tilde{r}}} d p_{\tilde{r}}^{\prime} d \tilde{r}
$$

where $p_{\tilde{r}}$ is the radial momentum, $\tilde{r}_{i}$ is the initial position, slightly outside the apparent horizon. And $\tilde{r}_{f}$ is a classical turning point, there the semiclassical trajectory can join onto a classical allowed motion. Furthermore, using the Hamlitonian equation,

$$
\dot{\tilde{r}}=\frac{\partial \tilde{H}}{\partial p_{\tilde{r}}}=\left.\frac{d \tilde{H}}{d p_{\tilde{r}}}\right|_{\tilde{r}},
$$

where $\tilde{H}$ is the Hamlitonian of the particle, the generator of the cosmic time $t$, we can carry out the integration in (15) as follows,

$$
\begin{aligned}
\operatorname{Im} \mathbf{S} & =\operatorname{Im} \int_{\tilde{r}_{i}}^{\tilde{r}_{f}} d \tilde{r} \int d \tilde{H} \frac{1}{\dot{\tilde{r}}} \\
& =\operatorname{Im} \int_{\tilde{r}_{i}}^{\tilde{r}_{f}} d \tilde{r} \frac{\omega}{\dot{\tilde{r}} \sqrt{1-k \tilde{r}^{2} / a^{2}}} \\
& =-\omega \operatorname{Im} \int_{\tilde{r}_{i}}^{\tilde{r}_{f}} \frac{d \tilde{r}}{\sqrt{1-k \tilde{r}^{2} / a^{2}}\left(\sqrt{H^{2} \tilde{r}^{2}+\left(1-\tilde{r}^{2} / \tilde{r}_{A}^{2}\right)}-H \tilde{r}\right)} \\
& =\pi \tilde{r}_{A} \omega
\end{aligned}
$$

Note that in de Sitter space case, the integration over the Hamiltonian $\tilde{H}$ simply gives the energy $\omega$ of the particle. In our case, it gives us $\omega / \sqrt{1-k \tilde{r}^{2} / a^{2}}$ since our energy of the particle is measured by an observer with the Kodama vector (7). Thus using the interpretation of emission rate (12), once again, we arrive at

$$
T=\frac{\omega}{2 \operatorname{ImS}}=\frac{1}{2 \pi \tilde{r}_{A}} .
$$

Thus we have shown again that the apparent horizon of FRW universe has an associated temperature, $1 / 2 \pi \tilde{r}_{A}$, like event horizon of black hole.

Conclusion and Discussions: In summary, by using the tunneling approach proposed by Parikh and Wilczek, we have finished the proof that the apparent horizon of FRW universe has indeed an associated Hawking temperature (2), filling in the gap existing in the literature. The Hawking temperature is measured by an observer with the Kodama vector (5) inside the apparent horizon. With this, we can conclude that Hawking radiation is not always associated with event horizon of spacetime. That is to say, the existence 
of event horizon is not a key cause of Hawking radiation, which was widely accepted before in the community of black hole physics. In addition, some remarks are in order. First, Hawking temperature is always related to surface gravity of horizon as $T=|\kappa| / 2 \pi$, where $\kappa$ is surface gravity of horizon. For the FRW universe, it is known that the surface gravity of apparent horizon is [15]: $\kappa=-\left(1-\dot{\tilde{r}}_{A} /\left(2 H \tilde{r}_{A}\right)\right) / \tilde{r}_{A}$. In deriving Friedmann equations by using $\delta Q=T d S$, a key point is to calculate the amount of energy crossing the apparent horizon in an infinitesimal time interval. During the infinitesimal time interval, the radius of the apparent horizon is assumed to be fixed, that is, $\dot{\tilde{r}}_{A}=0$. Thus one is led to $T=1 / 2 \pi \tilde{r}_{A}$. On the other hand, the tunneling process discussed in the present paper is an instantaneous one, one has naturally $\dot{\tilde{r}}_{A}=0$. Therefore we have the Hawking temperature (2) for the apparent horizon of FRW universe. In general, one believes there is a well-known relation between Hawking temperature and surface gravity for spacetime horizons, $T=|\kappa| / 2 \pi$. Therefore, it is of great interest to see the recovery of the relation by improving our discussions. Second, in the present paper, since did not consider the back-reaction of Hawking radiation, we therefore obtained an exact thermal spectrum of apparent horizon radiation. Naturally, if the back-reaction is taken into account, the radiation spectrum will deviate from the thermal spectrum. Since the deviation from the thermal spectrum of black hole is intensively discussed in the literature, we do not repeat here. Third, our proof can be easily generalized to the case of higher dimensional FRW universe with the same temperature (2). Finally we stress that although we get the Hawking temperature in the coordinates (44), one can reach the same conclusion starting with the metric (3) within the Hamilton-Jacobi method.

\section{Acknowledgments}

This work was supported partially by grants from NSFC, China (No. 10821504 and No. 10525060), and a grant from the Chinese Academy of Sciences with No.KJCX3-SYW-N2.

\section{References}

[1] S. W. Hawking, Commun. Math. Phys. 43, 199 (1975).

[2] J. D. Bekenstein, Phys. Rev. D 7, 2333 (1973).

[3] J. M. Bardeen, B. Carter and S. W. Hawking, Commun. Math. Phys. 31, 161 (1973).

[4] W. G. Unruh, Phys. Rev. D 14, 870 (1976). 
[5] G. W. Gibbons and S. W. Hawking, Phys. Rev. D 15, 2738 (1977).

[6] S. A. Hayward, R. Di Criscienzo, L. Vanzo, M. Nadalini and S. Zerbini, arXiv:0806.0014 [gr-qc]; R. Di Criscienzo, M. Nadalini, L. Vanzo, S. Zerbini and G. Zoccatelli, Phys. Lett. B 657, 107 (2007) [arXiv:0707.4425 [hep-th]].

[7] K. Srinivasan and T. Padmanabhan, Phys. Rev. D 60, 024007 (1999) arXiv:gr-qc/9812028; S. Shankaranarayanan, T. Padmanabhan and K. Srinivasan, Class. Quant. Grav. 19, 2671 (2002) arXiv:gr-qc/0010042; M. Angheben, M. Nadalini, L. Vanzo and S. Zerbini, JHEP 0505, 014 (2005) arXiv:hep-th/0503081.

[8] T. Jacobson, Phys. Rev. Lett. 75, 1260 (1995) arXiv:gr-qc/9504004.

[9] C. Eling, R. Guedens and T. Jacobson, Phys. Rev. Lett. 96, 121301 (2006) arXiv:gr-qc/0602001].

[10] M. Akbar and R. G. Cai, Phys. Lett. B 635, 7 (2006) arXiv:hep-th/0602156.

[11] M. Akbar and R. G. Cai, Phys. Lett. B 648, 243 (2007) arXiv:gr-qc/0612089.

[12] R. G. Cai and L. M. Cao, Phys. Rev. D 75, 064008 (2007) arXiv:gr-qc/0611071.

[13] E. Elizalde and P. J. Silva, arXiv:0804.3721 [hep-th].

[14] S. F. Wu, G. H. Yang and P. M. Zhang, arXiv:0805.4044 [hep-th].

[15] R. G. Cai and S. P. Kim, JHEP 0502, 050 (2005) arXiv:hep-th/0501055].

[16] A. V. Frolov and L. Kofman, JCAP 0305, 009 (2003) arXiv:hep-th/0212327; U. H. Danielsson, Phys. Rev. D 71, 023516 (2005) arXiv:hep-th/0411172; R. Bousso, Phys. Rev. D 71, 064024 (2005) arXiv:hep-th/0412197.

[17] S. A. Hayward, Class. Quant. Grav. 15, 3147 (1998) arXiv:gr-qc/9710089; S. A. Hayward, S. Mukohyama and M. C. Ashworth, Phys. Lett. A 256, 347 (1999) arXiv:gr-qc/9810006].

[18] M. Akbar and R. G. Cai, Phys. Rev. D 75, 084003 (2007) arXiv:hep-th/0609128.

[19] R. G. Cai and L. M. Cao, Nucl. Phys. B 785, 135 (2007) arXiv:hep-th/0612144; A. Sheykhi, B. Wang and R. G. Cai, Nucl. Phys. B 779, 1 (2007) arXiv:hep-th/0701198; A. Sheykhi, B. Wang and R. G. Cai, Phys. Rev. D 76, 023515 
(2007) arXiv:hep-th/0701261; R. G. Cai, L. M. Cao and Y. P. Hu, arXiv:0807.1232 [hep-th];

[20] R. G. Cai, Prog. Theor. Phys. Suppl. 172, 100 (2008) [arXiv:0712.2142 [hep-th]].

[21] X. H. Ge, Phys. Lett. B 651, 49 (2007) arXiv:hep-th/0703253; Y. Gong and A. Wang, Phys. Rev. Lett. 99, 211301 (2007) arXiv:0704.0793 [hep-th]]. S. F. Wu, G. H. Yang and P. M. Zhang, arXiv:0710.5394 [hep-th]. S. F. Wu, B. Wang and G. H. Yang, Nucl. Phys. B 799, 330 (2008) arXiv:0711.1209 [hep-th]; S. F. Wu, B. Wang, G. H. Yang and P. M. Zhang, arXiv:0801.2688 [hep-th]; T. Zhu, J. R. Ren and S. F. Mo, arXiv:0805.1162 [gr-qc]; M. Akbar, arXiv:0808.0169 [gr-qc].

[22] T. Padmanabhan, Class. Quant. Grav. 19, 5387 (2002) [arXiv:gr-qc/0204019]; T. Padmanabhan, Phys. Rept. 406, 49 (2005) arXiv:gr-qc/0311036]; T. Padmanabhan, Int. J. Mod. Phys. D 15, 1659 (2006) arXiv:gr-qc/0606061]; A. Paranjape, S. Sarkar and T. Padmanabhan, Phys. Rev. D 74, 104015 (2006) arXiv:hep-th/0607240]; D. Kothawala, S. Sarkar and T. Padmanabhan, Phys. Lett. B 652, 338 (2007) arXiv:gr-qc/0701002.

[23] M. K. Parikh and F. Wilczek, Phys. Rev. Lett. 85, 5042 (2000) arXiv:hep-th/9907001.

[24] M. K. Parikh, Phys. Lett. B 546, 189 (2002) arXiv:hep-th/0204107; A. J. M. Medved, Phys. Rev. D 66, 124009 (2002) arXiv:hep-th/0207247].

[25] H. Kodama, Prog. Theor. Phys. 63, 1217 (1980).

[26] S. A. Hayward, Phys. Rev. D 53, 1938 (1996) arXiv:gr-qc/9408002].

[27] C. W. Misner and D. H. Sharp, Phys. Rev. 136, B571 (1964). 